\title{
PALYNO-MORPHOLOGICAL STUDY OF WEEDY MELLIFEROUS (BEE VISITED) PLANTS USING LIGHT MICROSCOPIC TECHNIQUES FROM SOUTHERN KHYBER PAKHTUNKHWA, PAKISTAN
}

Khushdil Khan ${ }^{1}$, Mushtaq Ahmad ${ }^{1}$, Muhammad Zafar ${ }^{1}$, Khafsa Malik ${ }^{2}$, Shazia Sultana $^{1}$, Shabir Ahmad ${ }^{1}$, Fawad Khan ${ }^{3}$, Asif kamal' ${ }^{1}$ Kalim Ullah $^{3}$

DOI: https://doi.org/10.28941/pjwsr.v27i2.925

\begin{abstract}
Pollen morphology of 10 different weedy bee foraged plants belong to 10 various families from Southern Khyber Pakhtunkhwa were collected, identified, and studied using light microscopy (LM). The plants were Asphodelus tenuifolius, Euphorbia helioscopia, Parthenium hysterophorus, Rhazya stricta, Datura innoxia, Eruca sativa, Convolvulus arvensis, Anagallis arvensis, Galium aparine, and Anethum graveolens. Slides for light microscopic studies were prepared using materials of acetic acid, glycerin jelly and anthers of flowers. Pollen grain recorded ranged from monocolpate to hexacolporate and from psilate to echinate which were important systematic significance. Pollen size, shape, ratio of polar to equatorial diameter exine thickness, number of colpi, number of pores, equatorial diameter, polar diameter, colpus width, colpus length, spines number, length and width of spines were examined with the help of light microscopy and all these values were analyzed statistically using software SPSS. This research provides a data to the optimal utilization of bee foraged weed plants by honeybees and identification of bee flora for the beekeeping business and honey production. Results revealed that weedy melliferous flora of study area is very helpful for botanical origin, geographical origin of bee species and adulterations found in honey.
\end{abstract}

KEYWORDS: bee foraged, weeds, light microscopy, pollen, honey, beekeeping

Citation: Khan, K., M. Ahmad, M. Zafar, K.Malik, S. Sultana, S.Ahmad, F.Khan. 2021. Palynomorphological Study of Weedy Melliferous (Bee Visited) Plants Using Light Microscopic Techniques From Southern Khyber Pakhtunkhwa, Pakistan. Pak. J. Weed Sci. Res., 27 (2):163172.

${ }^{1}$ Department of Plants sciences, Quaid i Azam university, Islamabad.

2. Department of botany, Arid Agriculture University, Rawalpindi.

3. Department of botany, university of Peshawar, Pakistan.

Corresponding author: Email address: khushdilmarwat9090@gmail.com 


\section{INTRODUCTION}

Bee foraged plants is frequently visited by honeybees for nectars and pollen collections. The weedy melliferous flora are the honey source in southern Khyber Pakhtunkhwa. The study of weedy bee forage flora not limit to the field of taxonomy but also related to the field of plant genetics, plant biotechnology, plant ecology and molecular study of plants (Ahmad et al. 2019). Pollen is male gametophyte of bee foraged plants. Pollen morphological characteristics compared with other morphological properties of leaves, stems, roots, etc has high genetic stability. When honeybees visit the flowers to collect pollen, normally focus on only one bee foraged plant in each trip. Honeybee used the flower of weedy bee foraged flora as a source of food (Tashev and Pancheva, 2011). At some advanced level macro and micro morphological evolution of pollen has an important role in taxonomic studies( $\mathrm{Naz}$ et al. 2019). The honeybee is the primary pollinator of weedy melliferous flora both in wild and cultivated form ( Ponnuchamy et al. 2014). The pollen study of weedy bee foraged plants indicate about flora origin, medicinal and economics importance related to it (Saibal, 2005). Pollen study determines adulterations found in honey.

Plants is the natural source of honey, its floral parts suck by Honey bees to collect nectars and pollen ( Rosdi et al. 2016). Honey is sweet in tests and used as a medicine from millions of years. (Crane, 1975). Melissopalynology is an applicable field of pollen study. Bees collect pollen pellets, process it, store it in combination with certain substances and deposit in the comb cells (White and Doner, 1980). These pollen pellets also called as pollen load, bee meat or bee bread. In addition, pollen analysis provides important information about honey filtration, extraction, and fermentation (Russmann, 1998). Plant origin is determined according to the relative frequency of pollen species using melissopalynology ( Von Der Ohe et al. 2004). Weedy melliferous plants have colorful flowers, growing abundantly and blooming, which attract the bees for pollen, nectar, and honeydew for honey make up ( Hrynko et al. 2019). Fresh supply of nectars used to build storage combs in hive (Ramanujam and Kalpana, 1994). Therefore, weedy bee foraged plants is primary source for progress and growth of apiculture industry. The Southern Khyber Pakhtunkhwa is divided into districts, for example Lakki Marwat, Karak, Bannu, D I Khan (Akhtar et al., 2018).

Aim of the present study is to investigate pollen morphological study of weedy melliferous plants from southern Khyber Pakhtunkhwa while using microscopic techniques. The study also helps to examine the bee foraged plants in the study area, share the local knowledge regarding to beekeeping, availability of honey and determination of seasons for honey productions.

\section{MATERIAL \& METHODS SAMPLE COLLECTIONS}

A field survey was arranged during the start of February in Southern Khyber Pakhtunkhwa. All weedy bee foraged plants were collected from different areas of the Southern Khyber Pakhtunkhwa including Jathanbanda, Inzar, Wadinshaw, Seraj Khel, Khamedan, Amberikalai, Garang, Naraikhwara, Tati and Sabir Abad. All the 13 weedy bee foraged species collected from the study area belong to 11 different families including wild species. During the field trip before the collection of plants, we were confirmed that such weed plants is frequently visited by honeybees for pollen and nectars. After complete confirmation, plants were photographed, then collected with flowers and brought to the Lab for identification and research work.

\section{LIGHT MICROSCOPIC STUDIES}

First of all, from the anther of flower, the pollen grain was taken and then put on the slides for microscopic study. According to the techniques of acetolysis of Erdtman, the pollen grain was prepared for light microscopic study (Erdtman, 1969). Pollen was then treated 
with Acetic acid and crush with the help of a metal rod. As a result of these crushing's pollen released from the sac of stamen. All the debris because of crushing were discarded with a small camel brush and pure pollen grain treated with glycerin jelly for staining purposes. Glycerin jelly were prepared through method of (Meo and Khan, 2005). Coverslip was then put on the slide; readings of pollen were taken with the help of a microscope. Voucher number and taxon name were noted on the slide and then put in the slides box. All measurement of pollens were taken within 7 days. Fertile and sterile pollen numbers were counted in the slides with the help of Light Microscope (LM). We compile quantitative and qualitative data of pollen and show in the Table $1 \& 2$. In qualitative characters pollen size and shape were noted.
In quantitative characters, we studied about the equatorial diameter, polar diameter, colpi width, colpi length, colpi number, exine thickness, spine width and length with the help of Light Microscopy. Micrographs of pollen captured with the assistance of Leica magnifying lens fixed with camera Meiji infinity 1.

\section{RESULTS \& DISCUSSION.}

All the 13 weedy plants of 11 different families were clearly observed in the field as a melliferous character and studied its pollen with the help of light microscopic techniques. Both quantitative and qualitative character of pollen morphology including polar diameter, equatorial diameter, exine thickness, ratio of polar to equatorial diameter were showed in (Table $1 \& 2$ ). 


\begin{tabular}{|c|c|c|c|c|c|c|}
\hline S.No & Taxa & Collection site & $\begin{array}{l}\text { Vernacular } \\
\text { name }\end{array}$ & Family & $\begin{array}{l}\text { Cultivation status/ Distribution } \\
\text { within Pakistan }\end{array}$ & Distribution in World \\
\hline 1 & $\begin{array}{l}\text { Anagallis arvensis } \\
\mathrm{L} \text {. }\end{array}$ & $\begin{array}{l}\text { Amberi } \quad \text { kalai } \\
\text { (karak) }\end{array}$ & Warkharai & Primulaceae & $\begin{array}{l}\text { Sawabi, Charsada, Karak, Mardan, } \\
\text { Lakki Marwat, Bannu. Islamabad, } \\
\text { Rawalpindi, Swat, Jehlum, Kaghan, } \\
\text { Abbottabad, Nowshera. }\end{array}$ & $\begin{array}{l}\text { Mexico, Colombia, Venezuela, Brazil, } \\
\text { Sudan, Nigeria, china, Pakistan, South } \\
\text { east Asia, and North America. }\end{array}$ \\
\hline 2 & $\begin{array}{l}\text { Anethum } \\
\text { graveolens L }\end{array}$ & $\begin{array}{l}\text { Jathan banda } \\
\text { (karak) }\end{array}$ & Kalvangi & Apiaceae & $\begin{array}{l}\text { Mardan, lakki marwat, Bannu, } \\
\text { Karak, swat, Karachi, Islamabad, } \\
\text { Peshawar, Charsada, Lower and } \\
\text { Upper dir. }\end{array}$ & $\begin{array}{l}\text { Australia, Nepal, Bangladesh, Italy, } \\
\text { Africa, North America, Japan, South } \\
\text { west Asia, Afghanistan, Pakistan, } \\
\text { India. }\end{array}$ \\
\hline 3 & $\begin{array}{l}\text { Asphodelus } \\
\text { tenuifolius Cav. }\end{array}$ & Inzar (karak) & Pazakai & Asphodelaceae & $\begin{array}{l}\text { Bannu, Dera Ismail Khan, Tank, } \\
\text { Lakki Marwat, Karak, Nowshera, } \\
\text { Rawalpindi, Jhelum, Peshawar. }\end{array}$ & $\begin{array}{l}\text { North Africa, Europe, South West Asia, } \\
\text { Pakistan, India, Somalia, Asia, } \\
\text { Afghanistan. }\end{array}$ \\
\hline 4 & $\begin{array}{l}\text { Convolvulus } \\
\text { arvensis L. }\end{array}$ & Serak (karak) & Parkhatoon & $\begin{array}{l}\text { Convolvulacea } \\
\text { e }\end{array}$ & $\begin{array}{l}\text { Mardan, Sawabi, Mianwali, Karak, } \\
\text { Islam abad, Rawalpindi, Lahore, } \\
\text { Peshawar, Murree, Kaghan, Naran, } \\
\text { Swat. }\end{array}$ & $\begin{array}{l}\text { Asia, Europe, Maldives, Sri Lanka, } \\
\text { India, Pakistan, Bangladesh. }\end{array}$ \\
\hline 6 & $\begin{array}{l}\text { Eruca vesicaria } \\
\text { (L.) Cav. }\end{array}$ & Serak (karak) & Salaad & Brassicaceae & $\begin{array}{l}\text { Mardan, Sawabi, Lakki marwat, } \\
\text { Bannu, karak, Jehlum, Charsada, } \\
\text { Lower Dir, Rawalpindi. }\end{array}$ & $\begin{array}{l}\text { Mediterranean, North east Africa, } \\
\text { Australia, America, Singapore, china, } \\
\text { Pakistan, Russia, India, Center Asia. }\end{array}$ \\
\hline 7 & $\begin{array}{l}\text { Euphorbia } \\
\text { helioscopia L. }\end{array}$ & $\begin{array}{l}\text { Seraj } \\
\text { (karak) }\end{array}$ & Perparai & Euphorbiaceae & $\begin{array}{l}\text { Lakki marwat, Bannu, Bahawalpur, } \\
\text { Isa khel, Karak, Sawabi, } \\
\text { Mianwali,and South punjab }\end{array}$ & $\begin{array}{l}\text { America, Africa, Bhutan, Bangladesh, } \\
\text { Pakistan, Afghanistan, Sri Lanka. }\end{array}$ \\
\hline 8 & Galium aparine $\mathrm{L}$ & Tati (karak) & Awatunkai & Rubiaceae & $\begin{array}{l}\text { South Punjab, Bannu, lakki marwat, } \\
\text { Dera Ismail Khan, Karak, } \\
\text { Rawalpindi, Jhelum, Lahore, Ziarat, } \\
\text { Karachi, Hyderabad, Nowshera. }\end{array}$ & $\begin{array}{l}\text { India Japan, South west Asia, Nepal, } \\
\text { Bhutan, Bangladesh, North America, } \\
\text { and Brazil. }\end{array}$ \\
\hline 9 & $\begin{array}{l}\text { Parthenium } \\
\text { hysterophorus L. }\end{array}$ & $\begin{array}{l}\text { Amberikalai } \\
\text { (karak) }\end{array}$ & Spin gulai & Asteraceae & $\begin{array}{l}\text { Karak, Bannu, Swabi, Lakki } \\
\text { marwat, Dera Ismail Khan, Mardan, } \\
\text { Mianwali, Peshawar, Hazara, } \\
\text { Islamabad. }\end{array}$ & $\begin{array}{l}\text { Africa, Australia, Ethiopia, Africa, } \\
\text { Switzerland, Afghanistan, Pakistan, } \\
\text { Uganda. }\end{array}$ \\
\hline 10 & $\begin{array}{l}\text { Rhazya } \\
\text { Decne. }\end{array}$ & $\begin{array}{l}\text { Khamedan } \\
\text { (karak) }\end{array}$ & Zara wena & Apocynaceae & $\begin{array}{l}\text { Mardan, Mianwali, Karak, Lakki } \\
\text { Marwat, Dera Ismail Khan, Hazara, } \\
\text { Islam abad, Rawalpindi. }\end{array}$ & $\begin{array}{l}\text { Saudi Arabia, japan, America, Bhutan, } \\
\text { Maldeep, Afghanistan, } \\
\text { Bangladesh. }\end{array}$ \\
\hline
\end{tabular}

TABLE 1. Melliferous plant collection and their distribution 


\begin{tabular}{|l|l|l|l|l|l|l|l|}
\hline S.No & Taxa & Pollen shape & Colpi & Number of colpi & Spines & Shape of spines & Exine Sculpturing \\
\hline 1 & Anagallis arvensis L. & Oblate -spheroidal & P & Tricolporate & A & A \\
\hline 2 & Anethum graveolens L. & Prolate & P & P & A & A \\
\hline 3 & Asphodelus tenuifolius Cav. & Prolate -spheroidal & P & Monocolpate & A & A & - \\
\hline 4 & Convolvulus arvensis L. & Sub prolate & P & Tricolpate & A & A & - \\
\hline 5 & Datura innoxia Mill. & Sub oblate & P & Tricolpate & A & A & Psilate \\
\hline 6 & Eruca vesicaria (L.) Cav. & Oblate-spheroidal & P & Tricolpate & A & A & Psilate \\
\hline 7 & Euphorbia helioscopia L. & Oblate-spheroidal & P & Tricolpate & A & A & A \\
\hline 8 & Galium aparine L. & Prolate & P & Tricolporate & A & P & Small and cylindrical \\
\hline 9 & Parthenium hysterophorus L. & Oblate -spheroidal & P & Tricolpate & A & A \\
\hline 10 & Rhazya stricta Decne. & Sub prolate & P & Tricolpate & Psilate \\
\hline
\end{tabular}

TABLE 2. Micro-morphological pollen-Qualitative characters of melliferous flora.

Keywords: $P=$ Present, $A=$ Absent

\begin{tabular}{|c|c|c|c|c|c|c|c|c|c|}
\hline S.No & Species name & $\begin{array}{l}\text { P/E ratio } \\
\text { Mean } \\
(\min - \\
\max ) \quad S E \\
\mu \mathrm{m}\end{array}$ & $\begin{array}{l}\text { Exine } \\
\text { thickness } \\
\text { Mean (min- } \\
\text { max) SE } \mu \mathrm{m}\end{array}$ & $\begin{array}{l}\text { Polar diameter } \\
\text { Mean (min- } \\
\text { max) SE } \mu \mathrm{m}\end{array}$ & $\begin{array}{l}\text { Equatorial } \\
\text { diameter } \\
\text { Mean (min- } \\
\max \text { ) SE } \mu \mathrm{m}\end{array}$ & $\begin{array}{l}\text { Colpi length } \\
\text { Mean (min- } \\
\text { max) SE } \mu \mathrm{m}\end{array}$ & $\begin{array}{l}\text { Colpi width } \\
\text { Mean (min- } \\
\text { max) SE } \mu \mathrm{m}\end{array}$ & \begin{tabular}{|l} 
Spine \\
length \\
Mean \\
(min-max) \\
SE $\mu \mathrm{m}$ \\
\end{tabular} & $\begin{array}{l}\text { Spine } \\
\text { width } \\
\text { Mean } \\
\text { (min-max) } \\
\text { SE } \mu \mathrm{m}\end{array}$ \\
\hline 1 & Anagallis arvensis L. & 0.94 & $\begin{array}{l}1.80(1.70- \\
1.90) \\
\pm 0.03 \mathrm{~s}\end{array}$ & $\begin{array}{l}12.54(10.5- \\
14.50) \pm 0.75\end{array}$ & $\begin{array}{l}13.22(12.50- \\
14.00) \pm 0.25\end{array}$ & $\begin{array}{l}3.78(1.90-6.50) \\
\pm 1.11\end{array}$ & $\begin{array}{l}2.00(1.90- \\
2.10) \pm 0.03\end{array}$ & A & A \\
\hline 2 & Anethum graveolens L. & 1.58 & $\begin{array}{l}2.32(2.20- \\
2.40) \pm 0.03\end{array}$ & $\begin{array}{l}10.62(10.00- \\
11.10) \pm 0.20\end{array}$ & $\begin{array}{l}6.72(6.40- \\
7.10) \pm 0.13\end{array}$ & $\begin{array}{l}3.04(2.90-3.20) \\
\pm 0.05\end{array}$ & $\begin{array}{l}3.50(3.40- \\
3.60) \pm 0.03\end{array}$ & A & A \\
\hline 3 & Asphodelus tenuifolius Cav. & 1.02 & $\begin{array}{l}2.22(2.10- \\
2.22) \pm 0.04\end{array}$ & $\begin{array}{l}27.06(26.90- \\
27.06) \pm 0.07\end{array}$ & $\begin{array}{l}26.52(26.00- \\
26.52) \pm 0.27\end{array}$ & $\begin{array}{l}27.12(26.70- \\
27.12) \pm 0.16\end{array}$ & $\begin{array}{l}6.60(6.40- \\
6.60) \pm 0.07\end{array}$ & A & A \\
\hline 4 & Convolvulus arvensis L. & 1.32 & $\begin{array}{l}3.70(3.50- \\
4.10) \pm 0.12\end{array}$ & $\begin{array}{l}40.00(39.50- \\
41.00) \pm 0.27\end{array}$ & $\begin{array}{l}30.28(29.50- \\
31.00) \pm 0.25\end{array}$ & $\begin{array}{l}15.02(14.50- \\
15.50) \pm 0.16\end{array}$ & $\begin{array}{l}18.60(18.00- \\
19.00) \\
\pm 0.19\end{array}$ & A & A \\
\hline 5 & Datura innoxia Mill. & 0.81 & $\begin{array}{l}1.76(1.70- \\
1.80) \pm 0.02\end{array}$ & $\begin{array}{l}13.66(13.40- \\
14.00) \pm 0.11\end{array}$ & $\begin{array}{l}16.72(16.50- \\
17.00) \pm 0.10\end{array}$ & $\begin{array}{l}16.46(15.70- \\
16.90) \pm 0.20\end{array}$ & $\begin{array}{l}3.68(3.50- \\
3.90) \pm 0.07\end{array}$ & A & A \\
\hline 6 & Eruca vesicaria (L.) Cav. & 0.92 & $\begin{array}{l}1.76(1.70- \\
1.90) \pm 0.04\end{array}$ & $\begin{array}{l}11.04(10.60- \\
11.60) \pm 0.16\end{array}$ & $\begin{array}{l}11.96(11.50- \\
12.70) \pm 0.20\end{array}$ & $\begin{array}{l}5.62(5.50-5.80) \\
\pm 0.06\end{array}$ & $\begin{array}{l}5.86(5.70- \\
6.10) \pm 0.08\end{array}$ & A & A \\
\hline
\end{tabular}




\begin{tabular}{|c|c|c|c|c|c|c|c|c|c|}
\hline 7 & Euphorbia helioscopia L. & 0.97 & $\begin{array}{l}2.72(2.50- \\
2.90) \pm 0.07\end{array}$ & $\begin{array}{l}18.68(18.30- \\
19.10) \pm 0.16\end{array}$ & $\begin{array}{l}19.12(18.90- \\
19.50) \pm 0.10\end{array}$ & $\begin{array}{l}4.52(4.40-4.60) \\
\pm 0.04\end{array}$ & $\begin{array}{l}5.74(5.50- \\
6.00) \pm 0.09\end{array}$ & A & A \\
\hline 8 & Galium aparine L. & 1.35 & $\begin{array}{l}2.10(2.00- \\
2.30) \pm 0.54\end{array}$ & $\begin{array}{l}14.72(14.00- \\
15.10) \pm 0.19\end{array}$ & $\begin{array}{l}10.90(10.50- \\
11.20) \pm 0.13\end{array}$ & $\begin{array}{l}6.78(6.50-7.20) \\
\pm 0.15\end{array}$ & $\begin{array}{l}4.66(4.50- \\
5.00) \pm 0.92\end{array}$ & A & A \\
\hline 9 & $\begin{array}{l}\text { Parthenium hysterophorus } \\
\text { L. }\end{array}$ & 0.96 & $\begin{array}{l}2.40(2.30- \\
2.50) \pm 0.04\end{array}$ & $\begin{array}{l}7.68(7.50- \\
7.90) \pm 0.08\end{array}$ & $\begin{array}{l}7.96(7.70- \\
8.20) \pm 0.08\end{array}$ & A & $\mathrm{A}$ & $\begin{array}{l}2.76(2.50- \\
3.10) \\
\pm 0.10\end{array}$ & \begin{tabular}{|l|}
$1.80(1.60-$ \\
$2.10)$ \\
\pm 0.08
\end{tabular} \\
\hline 10 & Rhazya stricta Decne. & 1.30 & $\begin{array}{l}2.12(1.90- \\
2.40) \pm 0.09\end{array}$ & $\begin{array}{l}23.84(22.50- \\
25.00) \pm 0.39\end{array}$ & $\begin{array}{l}18.20(17.50- \\
19.00) \pm 0.24\end{array}$ & $\begin{array}{l}8.00(7.70-8.20) \\
\pm 0.09\end{array}$ & $\begin{array}{l}9.70(11.90- \\
12.00) \\
\pm 1.90\end{array}$ & A & A \\
\hline
\end{tabular}

TABLE 3. Micro-morphological pollen-Quantitative characters of melliferous flora.

Keywords: $A=$ absent; $\max =$ maximum; $\min =$ minimum; $S E=$ Standard error $; \mu \mathrm{m}=$ micrometer 


\section{Asphodelus tenuifolius $\mathbf{L}$}

Family: Asphodelaceae

English name: Asphodelus

Local name: Pazakai

Location: Inzar

Colour of Flower: White

Pollen Morphology: Pollen is monocolporate and monad. Pollen diameter in polar case is $27.06 \mu \mathrm{m}$ $(26.90-27.06 \mu \mathrm{m})$, equatorial diameter is $26.52 \mu \mathrm{m} \quad(26.00-26.52 \mu \mathrm{m}), \quad \mathrm{P} / \mathrm{E} 1.02$ and exine thickness is $2.22 \mu \mathrm{m}$ (2.10$2.22 \mu \mathrm{m})$.

\section{Euphorbia helioscopia L.}

Family: Euphorbiaceae

English name: Sun spurge

Local name: Perparai

Location: Seraj Khel

Colour of Flower: Yellow

Pollen Morphology: Pollen is tricolporate and monad. Pollen diameter in polar case is $18.68 \mu \mathrm{m} \quad(18.30-19.10 \mu \mathrm{m})$, equatorial diameter is $19.12 \mu \mathrm{m}$ (18.90$19.500 \mu \mathrm{m}), P / E$ is 0.97 and exine thickness is $2.72 \mu \mathrm{m}(2.50-2.90 \mu \mathrm{m})$.

\section{Parthenium hysterophorus $\mathbf{L}$}

Family: Asteraceae

English name: Carrot grass

Local name: Spin gulai

Location: Amberikalai

Colour of Flower: White

Pollen Morphology: Pollen is tricolporate, echinate and monad. Polar diameter of pollen is $7.68 \mu \mathrm{m} \quad(7.50-7.90 \mu \mathrm{m})$, equatorial diameter is $7.96 \mu \mathrm{m}$ (7.70$8.20 \mu \mathrm{m}), \mathrm{P} / \mathrm{E}$ ratio is 0.96 and exine thickness is $7.96 \mu \mathrm{m}(7.70-8.20 \mu \mathrm{m})$.

\section{Rhazya stricta Decne}

Family: Apocynaceae

English name: Harmal

Local name: Zara wena

Location: Khamedan

Colour of Flower: White

Pollen Morphology: Pollen is tricolporate and monad. Polar diameter of pollen is $23.84 \mu \mathrm{m} \quad(22.50-25.00 \mu \mathrm{m})$, equatorial diameter is $18.20 \mu \mathrm{m}(17.50-19.00 \mu \mathrm{m})$, $\mathrm{P} / \mathrm{E}$ is 1.30 and exine thickness is $2.12 \mu \mathrm{m}(1.90-2.40 \mu \mathrm{m})$.

\section{Datura innoxia Mill}

Family: Solanaceae

English name: Hoary thorn apple

Local name: Rhand

Location: Khamedan

Colour of Flower: White
Pollen Morphology: Pollen is tricolporate and monad. Polar diameter of pollen is $13.66 \mu \mathrm{m} \quad(13.40-14.00 \mu \mathrm{m})$, equatorial diameter is $16.72 \mu \mathrm{m}(16.50-17.00 \mu \mathrm{m})$, $\mathrm{P} / \mathrm{E}$ is 0.81 and exine thickness is $1.98 \mu \mathrm{m}(1.90-2.10 \mu \mathrm{m})$.

\section{Eruca sativa Mill}

Family: Brassicaceae

English name: Rocket salad

Local name: Salad

Location: Serak

Colour of Flower: White -Yellow

Pollen Morphology: Pollen is tricolporate and monad. Polar diameter is $11.04 \mu \mathrm{m}$ $(10.60-11.60 \mu \mathrm{m})$, equatorial diameter is $11.96 \mu \mathrm{m}(11.50-12.70 \mu \mathrm{m}), \mathrm{P} / \mathrm{E}$ is 0.92 and exine thickness is $1.76 \mu \mathrm{m}$ (1.70$1.90 \mu \mathrm{m})$.

\section{Convolvulus arvensis $\mathbf{L}$}

Family: Convolvulaceae

English name: Chardvel

Local name: Parkhatoon

Location: Serak

Colour of Flower: White

Pollen Morphology: Pollen is tricolporate and monad. Polar diameter of pollen is $40.00 \mu \mathrm{m} \quad(39.50-41.00 \mu \mathrm{m})$, equatorial diameter is $30.28 \mu \mathrm{m}(29.50-31.00 \mu \mathrm{m})$, $\mathrm{P} / \mathrm{E}$ is ratio is 1.32 and exine thickness is

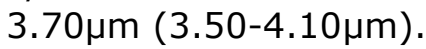

\section{Anagallis arvensis $\mathbf{L}$.}

Family: Primulaceae

English name: Red scarlet

Local name: Warkharai

Location: Ambari Kala

Colour of Flower: Bluish

Pollen Morphology: Pollen is monad with 9 colpi and 9 pores, Polar diameter is $14.72 \mu \mathrm{m}$ (14.00-15.10 $\mu \mathrm{m})$, equatorial diameter is $10.90 \mu \mathrm{m}(10.50-11.20 \mu \mathrm{m})$, $\mathrm{P} / \mathrm{E}$ is 1.35 and exine thickness is $2.10 \mu \mathrm{m}(2.00-2.30 \mu \mathrm{m})$.

\section{Galium aparine $\mathrm{L}$.}

Family: Rubiaceae

English name: Cleavers

Local name: Awatunkai

Location: Tati

Flower color: White

Pollen Morphology: Pollen is tricolporate and monad. Polar diameter is $12.54 \mu \mathrm{m}$ $(10.5-14.50 \mu \mathrm{m})$, equatorial diameter is $13.22 \mu \mathrm{m}(12.50-14.00 \mu \mathrm{m}), \mathrm{P} / \mathrm{E}$ is 0.94 and exine thickness is $1.80 \mu \mathrm{m}$ (1.70$1.90 \mu \mathrm{m})$. 
10. Anethum graveolens $\mathbf{L}$

Family: Apiaceae

English name: Sanskrit

Local name: Kalvangi

Location: Jathan banda

Colour of Flower: Yellow

Pollen Morphology: Pollen is monad and dicolporate. Polar diameter is $10.62 \mu \mathrm{m}$ $(10.00-11.10 \mu \mathrm{m})$, equatorial diameter is $6.72 \mu \mathrm{m}(6.40-7.10 \mu \mathrm{m}), \mathrm{P} / \mathrm{E}$ is ratio 1.58 and exine thickness is $2.32 \mu \mathrm{m}$ (2.20$2.40 \mu \mathrm{m})$.

In bee foraged plants we observed three groups (i) pollen source, which is visited for pollen collection (ii) nectars source, which is visited for nectars collection (iii) nectars and pollen source, which is visited for both nectars and collection for honey make up (Kalpana et al. 1990). These groups were confirmed from melissopalynology of different honey samples of the studied areas. Palynological study of investigated taxa was observed under the light microscope (LM); most of the pollen were tricolporate, and few are monocolporate and dicalporate. We measured different aspects of studied weedy bee foraged plant species as equatorial diameter, polar diameter, colpi length, colpi width, exine thickness, spines, and pores numbers. Differences in exine thickness of pollen was also examined in the reported study of (Asmat et al. 2011). The pollen and nectars collected by Apis mellifera from different weedy bee foraged vegetation as shrub, and herbs. The maximum $P / E$ ratio was observed in Anethum graveolens was $1.58 \mu \mathrm{m}$. The minimum P/E ratio was noted in Datura innoxia Mill. $0.81 \mu \mathrm{m}$. Maximum exine thickness was observed in Convolvulus arvensis as $3.70 \mu \mathrm{m}$ while the minimum one was $1.76 \mu \mathrm{m}$ noted in Datura innoxia. The Convolvulus arvensis was maximum polar diameter as $40.00 \mu \mathrm{m}$ while the minimum polar diameter was noted in Parthenium hysterophorus as $7.68 \mu \mathrm{m}$. The maximum equatorial diameter was noted in Convolvulus arvensis as $30.28 \mu \mathrm{m}$ while minimum observed in Anethum graveolens as 6.72 $\mu \mathrm{m}$. The maximum colpi length was observed in Asphodelus tenuifolius is 27.12. $\mu \mathrm{m}$ while minimum observed in Anethum graveolens is $3.04 \mu \mathrm{m}$. The studied plants show maximum Spine length of Sonchus asper is $3.60 \mu \mathrm{m}$ while minimum in Parthenium hysterophorus is $2.76 \mu \mathrm{m}$. Convolvulus arvensis have maximum width of colpi is $18.60 \mu \mathrm{m}$ and Anagallis arvensis have minimum is $2.00 \mu \mathrm{m}$.

Most of the weedy bee foraged plants species origin are tropical dry ever green ; supported by the pollen used in the honey make up indicate the tropical dry Evergreen forest species ( Ponnuchamy et al. 2014). The studied plants blooming period show variation to species. The study of weedy bee foraged plants is help full for beekeeper to manage their business according to the flowering season in different areas (Noor et al, 2017). The melliferous weed plants are most important because local people conserve it for honey production. During field trip and interviews from local beekeepers, it is observed that in Southern Khyber Pakhtukhwa the dominant flowering period of melliferous weeds plants in March and April. The information about bee foraged species identifies botanical source of honey. Data of bee foraged plants can encourage the beekeeper for the beekeeping and maximize the honey production for export purposes to the developed countries in many areas (Bhalchandra et al. 2014). Endangerments of weedy bee foraged flora may cause to decrease in nutrition and honey production which causes diseases (Basu and Cetzal-Ix., 2018). Pollination in weedy melliferous flora mostly occurred by the entomophilous. In field observation the most common pollinators were Apis mellifera.

\section{CONCLUSION}

Weedy Bee foraged species were identified for managing bee keeping business and honey production. Pollen morphological studies of weedy bee foraged plants in the study area showing high potential of bee keeping business. The study area showed high potential of bee keeping business. In Southern Khyber Pakhtunkhwa purchase and sale of honey needs to be expended as well as need of quality control to identify non-Southern Khyber Pakhtunkhwa honey samples. Established some 
geographical limitation of best honey samples through adulteration detection. Pollen identification was possible with the help of previous published data. These results also confirm that Southern Khyber Pakhtunkhwa is main region for honey production due to presence of weedy bee foraged flora. The information's about weedy bee foraged flora may encouraged and propagate to development of bee forming in Southern Khyber Pakhtunkhwa, Pakistan.

\section{Literature cited.}

Ahmad, S., M. Zafar, M. Ahmad, G. Yaseen and S. Sultana. 2019. Microscopic investigation of Palynomorphological features of Melliferous flora of Lakki Marwat district, Khyber Pakhtunkhwa, Pakistan. Microsc. Res. Tech, 82(6), 720-730.

Akhtar, N., A. Siddique and M. Anwar. 2018. Angiospermic Plant resources and folk uses in District Karak, Khyber Pakhtunkhwa, Pakistan. JAPS: Journal of Animal \& Plant Sciences, 28(5) p1418-1418.

Asmat, T., M. A. Khan, M. Ahmed, M. Zafar, F. Manzoor, M. Munir and M. Ambreen. 2011. Pollen Morphology of selected species of Scrophulariaceae of District Dir Upper, Pakistan. J. Med. Plant Res. (28), 6423-6428.

Basu, S. K., and W. Cetzal-Ix. 2018. Call of the wild: Conservation of Natural insect Pollinators should be a priority. Biodiversity, 19(3-4), 240243.

Bhalchandra, W., R. Baviskar and T. Nikam. 2014. Diversity of Nectariferous and Polleniferous bee flora at Anjaneri and Dugarwadi hills of Western Ghats of Nasik District (MS) India. J. Entomol. Zool. Stud. 2(4), 244-249.

Crane, E., 1975. Honey: a Comprehensive Survey.

Erdtman, G. 1969. Handbook of palynology: Morphology, Taxonomy, Ecology.

Hrynko, I., B. Łozowicka, and P. Kaczyński. 2019. Comprehensive Analysis of insecticides in Melliferous weeds and Agricultural Crops using a Modified QuEChERS/LC-MS/MS Protocol and of their Potential risk to

\section{ACKNOWLEDGMENTS}

We are grateful to HEC, Pakistan for their monetary help under project no. $\mathrm{HEC} / \mathrm{R} \& \mathrm{D} / \mathrm{NRPU} / 2017 / 8420$. We are likewise grateful to the Lab of Plant Systematics and Biodiversity, QAU, Islamabad for giving research equipment's to complete all the experimental works.

Honey Bees (Apis mellifera L.). Sci. Total Environ. 657, 16-27.

Kalpana, T., K. Fatima, and C. Ramanujam. 1990. Pollen analysis of apis cerana and apis florea honeys from Adikmet area, Hyderabad. proceeding. indian academy of science 100:183-193.

Meo, A. A., and M. A. Khan. 2005. Pollen Morphology of invasive Species Parthenium hysterophorus L. (Heliantheae-Asteracea) from Islamabad and Rawalpindi, Pakistan. Sarhad Journal of Agriculture (Pakistan).

Naz, S., M. Zafar, M. Ahmad, R. A. Memon, S. Sultana, S. Bahadur, and M. A. Shah 2019. Palynological investigation of lactiferous flora (Apocynaceae) of District Rawalpindi, Pakistan, using light and Scanning Electron Microscopy. Microsc. Res. Tech. 82(9), 1410-1418.

Noor, M. J., M. Ahmad, M. Zafar, and M. A. Ashraf. 2017. Palynological studies of Melliferous and Allergenic Flora of Pakistan: A key to pollen and spore identification. J. Apic. Res. 56(3), 300-309.

Ponnuchamy, R., V. Bonhomme, S. Prasad, L. Das, P. Patel, C. Gaucherel and K. Anupama. 2014. Honey Pollen: using Melissopalynology to understand foraging preferences of Bees in Tropical South India. PloS one 9(7), e101618.

Rosdi, I. N., K. Selvaraju, P. Vikram, K. Thevan, and M. Arifullah. 2016. Melissopalynological Analysis of Forest Honey from North Malaysia. J. Trop. Resour. Sustain. Sci, 4, 128132. 
Rüssmann, H., Shams, H., Poblete, F., Fu, Y., Galán, J. E., \& Donis, R. O. (1998). Delivery of epitopes by the Salmonella type III secretion system for development. Science, 281(5376), 565-568.

Ramanjum, C., T. Kalpana. 1994. Summer pollen source for honey bees in the coastal belt of east Godaveri District, Andhra Pradesh, and india. Geophytology 23:249-252.

Saibal, C. 2005. Azadirachta indica L.-an important source of nectar and pollen in Nadia District of West Bengal. J. Phytol, 18(2), 179-182.
Tashev, A., and E. Pancheva. 2011. The Melliferous Plants of the Bulgarian Flora-Conservation importance. Forestry, 17(2), 42.

Von, D. O. W., L. P. Oddo, M. L. Piana, M. Morlot, and P. Martin. 2004. Harmonized Methods of Melissopalynology. Apidologie, 35(Suppl. 1), S18-S25.

White, J., and L.W. Doner. 1980. Honey Composition and properties. Beekeeping in the United States Agriculture Handbook, 335, 82-91.

Figure caption 1.pollen pictures (a) Anagallis arvensis L. (b) Anethum graveolens L. (c) Asphodelus tenuifolius Cav. (d) Convolvulus arvensis L. (e) Datura innoxia Mill. (f) Eruca vesicaria (L.) Cav. (g) Euphorbia helioscopia L. (h) Galium aparine L. (i) Parthenium hysterophorus L. (j) Rhazya stricta Decne.
